Natika Newton, Foundations of Understanding. (John Benjamins, 1996). 210 pages, $\$ 34.95$.

Natika Newton in Foundations of Understanding has given us a powerful, insightful and intriguing account of the experience of understanding. She offers compelling arguments for a foundationalist and internalist theory of understanding and intentionality, offering empirical evidence from the neurosciences to support her view. Although she is working within the analytic tradition, she sees a kinship between her theory and the work of the continental phenomenologists. This is so because she rejects the prevailing view among analytic philosophers of mind that cognition is best explained in terms of a linguistic paradigm. She contends that at the foundational level conscious understanding and conscious intentionality are best explained in terms of bodily experience rather than in terms of language or language-like structures. She joins a growing number of analytic philosophers who focus, as the phenomenologists earlier in the century did, on the embodied nature of consciousness. Her view is most closely related to that of Merleau-Ponty because like him she sees bodily intentionality as the most primary level of intentionality. But grounding cognition in action and grounding language in the non-linguistic also unites her view with that of the later Wittgenstein. Because of this dual kinship, Newton helps bridge the gap between continental and analytic philosophy. Her work should be of interest to philosophers from both traditions as well as those who believe a dialogue between the two traditions is important for furthering our philosophical understanding.

Newton's theory is essentially a theory of our experience of conscious understanding. It is a foundationalist theory because she argues that all experiences of understanding are derived from experiences of understanding our own basic voluntary actions. It is an internalist theory because, on her view, the conscious understanding of actions is constituted by something internal to the subject: reactivated sensorimotor experiences. Newton's theory is also a materialist one because she offers an account of understanding intentionality in terms of physical mechanisms: sensorimotor states.

\title{
The theory
}

Her plan for the book, she says in the introduction, is to analyze cases of conscious understanding and discover the mechanisms that make them possible. She believes this analysis of understanding will shed light on the nature of consciousness and intentionality as well. Because any intentional state is by nature about something, its existence requires an understanding of the something which the state is about. Hence an account of conscious understanding con- 
tributes to an account of conscious intentionality. Such an account contributes to an account of consciousness as well, since, if it is successful, it explains at least one type of conscious mental state.

The sensorimotor theory of cognition

Newton adheres to what she calls the sensorimotor theory of cognition, the belief that all higher cognition relies on the same structures as those used in sensorimotor activity. She combines this theory with her own version of a mental models approach to cognition. Her idea is that cognition involves the construction of analog models of reality and these models are constructed using representations that are sensorimotor images. Thinking involves the manipulation of these images not the manipulation of syntactic structures. To avoid construing Newton's theory as a kind of disguised Humean empiricism where thinking is the association of visual or quasi-visual pictures, the reader must pay careful attention to her repeated warnings that she is speaking of images in all sensory modalities not just the visual. In addition these images are not something that exist in some private mental sphere but are "the reactivation of past sensorimotor experiences of the subject" (p. 19). They are bodily experiences. In chapter two she offers a great deal of fascinating empirical evidence, behavioral, neuroscientific and evolutionary, to support her view that cognitive abilities are rooted in motor abilities. The remainder of the book offers an account of one primary cognitive activity: understanding and how understanding is rooted in motor activity.

\section{Understanding actions}

For Newton all understanding is founded in the nonconceptual understanding a person has of her own basic, goal-directed action. Such actions are understood in themselves and rely on no more basic understanding. This is so because the minimal condition for understanding a simple action is being "able to imagine performing the action, with an image rich enough to serve as a guide in the actual performance" of the action (p. 71). Such imagining involves the reactivation of sensorimotor experiences, but it does not involve the use of concepts or language which would themselves require further understanding. That is why, at its most primitive level, the understanding of actions (more accurately, action types) is the foundation of all understanding. Newton goes on in subsequent chapters to explain how the understanding of objects rests on the understanding of actions and how the understanding of the concept of a person rests on understanding of both objects and actions. In one of the last chapters, she argues that the understanding of language rests on all the subsequent forms of understanding. A hierarchy of types of understanding is developed with the foundation being the understanding of actions. 
The value of the theory

Newton offers us a plausible science and phenomenology based theory of understanding which founds understanding in the body and its structures. These are structures we share with human infants as well as with some nonhuman animals. Hence she provides us with a theory which allows for the existence of understanding in infants and other mammals and for a continuity between those forms of understanding and more sophisticated forms of understanding present in adult humans. In addition she offers us an alternative to the computational accounts of cognition with their inherent dualism and disregard for the body and its existence in the world. Because all forms of understanding are explained in terms of one basic form of understanding, Newton's theory has an inherent simplicity. In addition it avoids any threat of an infinite regress because the foundational form of understanding requires no other understanding to understand it. Newton also appeals to a wide range of scientific evidence which does not prove the theory is correct (and she never claims it does), but it does add to its plausibility.

One might ask whether Newton's foundationalist theory of understanding runs into the same kind of problems that haunt a Cartesian foundationalist epistemology which also finds the foundations in the inner world of the subject. I think it is a strength of the theory that the answer is no. Newton manages to give us an internalist account of understanding and intentionality which avoids the solipsism and the irreparable split between the self and the world that usually accompanies such theories. She does so by arguing that the imagery which is constitutive of understanding is a bodily state, which is, in principle, publicly accessible and by arguing that conscious experience arises from the blending of internal and external input. Hence experience unites rather than separates the self and the world.

\section{A problem for the theory}

Newton characterizes understanding as the reactivation of sensorimotor imagery. When one is conscious of the sensorimotor imagery, then one feels one understands; one is aware of understanding. Newton acknowledges, however, that it is possible for a person to understand something without the feeling of understanding being experienced. But why does it often appear that she conflates understanding and the feeling one understands? Why does she sometimes talk about her theory as a theory of understanding and at other times as a theory of the experience of understanding? I think this is because although one could understand without the conscious experience or feeling of understanding, the feeling when present is created by the consciousness of what Newton contends constitutes understanding - sensorimotor imagery. An analysis of the experience of understanding will, of course, include an analysis of 
the imagery which creates this feeling. It follows that given these characterizations of understanding and of the experience of understanding, an analysis of the experience will provide an analysis of understanding itself, whether the understanding is conscious or not. This is so because such an analysis will include a discussion of the sensorimotor imagery which, on her view, constitutes understanding. But Newton's analysis of the experience of understanding only works as an analysis of understanding itself if one accepts her contention that the feeling that one understands is always accompanied by the understanding of some sort even if such understanding is, to use her word, incorrect. But I think her assertion that she is not using the term understanding in a normative sense and so there can be understanding which is misunderstanding is misguided. It is part of the very meaning of the term that it is normative.

Incorrect understanding is not understanding of the wrong sort; it is not understanding at all. Her account of the feeling of understanding and its source in sensorimotor imagery is powerful and insightful and does indeed help to illuminate the nature of intentionality and consciousness. But her theory fails to capture the distinction between understanding and misunderstanding. What distinguishes a case where I feel I understand and I do not from one in which I feel I understand and I actually do? An account of the experience of understanding (thinking one understands) won't answer this question since the feeling is present in both cases. The presence or absence of sensorimotor imagery won't either since that is present in both cases according to Newton. The only way to capture the distinction between understanding and misunderstanding is to modify Newton's notion of what constitutes understanding. She contends that understanding is the reactivation of sensorimotor imagery. I would argue only the reactivation of the correct sensorimotor imagery constitutes understanding. If you accept her characterization of understanding, then you will believe, as she does, that cases of misunderstanding are, in some sense, cases of understanding, since sensorimotor imagery is present. But if the definition of understanding is modified in the way I suggest, then there is no need to deny the inherently normative nature of understanding. On the modified version of her theory understanding is still constituted by a bodily state, so an internalist theory of understanding is still possible if this modified version is correct. But knowing that one understands requires appeal to an objective world. This fits well with the explanation of error Newton offers in chapter five. This modified version explains how it is possible for one to understand without realizing one does. One does not, as Newton suggests, become aware that one understands simply by becoming conscious of the sensorimotor imagery. To be certain one understands one must establish that the imagery that has been reactivated matches the input from the external world and hence the external world itself. 
Newton herself raises more obvious problems with the theory. One such problem is how a theory which equates understanding with imagery can account for our understanding of abstract ideas. Another is that if imagery constitutes understanding, why do some people claim that although they are conscious of understanding, they are not conscious, even upon reflection, of the presence of the imagery she contends constitutes understanding. In her book, Newton offers convincing solutions to these problems.

In sum. Foundations of Understanding is an exciting exploration of human cognition. It takes the insights of earlier philosophers from both the analytic and continental traditions and extends and grounds them by appeal to current scientific findings. It does so without ever forgetting the bodily nature of the subject who understands.

University of Michigan-Dearborn

Kathleen Wider

Calvin O. Schrag, The SelfAfter Postmodernity. (New Haven and London: Yale University Press, 1997). Xiv + 155 pp.

This volume presents the revised text of the Gilbert Ryle Lectures given by the author at Trent University, Ontario, in 1995. It is commendable that the sponsors of the Ryle lectures have not limited themselves to philosophers of the analytic tradition, in which Ryle played such an important role, choosing on this occasion a distinguished representative of the Continental tradition. But we are living in a time when that distinction has in any case become outmoded, and as we look back, as Calvin Schrag points out here (pp. xif), we may be able to see Ryle in a different light. It is well known that in his early years he reviewed Heidegger for Mind, giving the German philosopher a respectful if not uncritical reading. He reviewed other phenomenological writers as well. More importantly, his attack on the mind-body split may be read, together with the work of Merleau-Ponty and others, as part of a common 20th century effort to get beyond this aspect of the Cartesian legacy.

As if in recognition that the analytic-continental divide is no longer a burning issue, Schrag, ever a conciliatory thinker, has little to say about it. Though he mentions Ryle a few times, his reconciling attentions are directed to another and more recent rift, this one within the tradition of continental philosophy itself. Continuing the project he began in his Communicative Praxis and the Space of Subjectivity (1986) and continued in The Resources of Rationality: A Response to the Postmodern Challenge (1992, both Indiana University Press), Schrag is concerned here with the post-modem turn in recent 